\title{
Developing a Point-of-Care Molecular Test to Detect SARS-CoV-2
}

\author{
Debjani Paul ${ }^{1}\left[\right.$ P Priyanka Naik ${ }^{1} \cdot$ Shomdutta Roy ${ }^{1}$
}

Received: 15 April 2020 / Revised: 5 June 2020 / Accepted: 10 June 2020 / Published online: 19 June 2020

(C) Indian National Academy of Engineering 2020

\begin{abstract}
There is a need for widespread testing in India to stop the spread of the novel coronavirus in the population. While RT-PCR is the recommended diagnostic technique, its use is limited to well-equipped laboratories due to the need for specialized instrumentation, reagents and trained personnel. Immunodiagnostic tests are not yet recommended by the WHO for diagnosing active infections. There is a strong need for developing point-of-care molecular tests. Based on our past experience with paperfluidic devices for diagnosing bacterial infections by molecular tests, we propose the development of a diagnostic test for COVID-19. As a platform technology, it could be adapted to other viral outbreaks in future.
\end{abstract}

Keywords Molecular diagnostics $\cdot$ Point-of-care test $\cdot$ Isothermal amplification $\cdot$ Paperfluidics

\section{The Problem with COVID-19 Diagnostics}

The recent pandemic of COVID-19 caused by the novel coronavirus (SARS-CoV-2) has drawn attention to the need for developing rapid and accurate diagnostic tests. The strategy to control the spread of the virus, as advised by the World Health Organization (WHO), requires testing of all suspected individuals, isolating them if they test positive and then tracing all their contacts to repeat the process. To implement this strategy in India, we need to massively scale up our testing capabilities. The test that is used to diagnose COVID-19 uses real-time reverse transcription polymerase chain reaction (real-time RT-PCR) to identify specific sequences of the viral RNA. Due to the need for expensive instrument, reagents and trained personnel to correctly perform RT-PCR, this test has been limited to well-equipped diagnostic and research laboratories.

Rapid immunodiagnostic tests can detect either antigens (i.e., viral proteins that are found in the host when the virus is actively replicating) or antibodies (i.e., host proteins developed in response to the virus). The WHO has issued an advisory on 8th April 2020 (https://www.who.int/ news-room/commentaries/detail/advice-on-the-use-of-point

Debjani Paul

debjani.paul@iitb.ac.in

1 Department of Biosciences and Bioengineering, Indian Institute of Technology Bombay, Powai, Mumbai 400076, India -of-care-immunodiagnostic-tests-for-covid-19) recommending the use of antibody tests only for understanding the spread of the pandemic and not for diagnosing active infections. This is because IgM and IgG antibodies in response to SARS-CoV-2 are found in blood only in the second week after the symptoms are manifested. Since this disease has a long incubation time (2-14 days), an antibody test is very likely to miss early infections. It should be noted that individuals in this early stage are capable of infecting others even if they do not manifest any symptoms. In contrast, an antigen-based test could potentially be used to diagnose pre-symptomatic individuals. We quote verbatim from the WHO's advice on antigen tests: "How well the tests work depends on several factors, including the time from onset of illness, the concentration of virus in the specimen, the quality of the specimen collected from a person and how it is processed, and the precise formulation of the reagents in the test kits (https://www.who.int/news-room/commentaries/ detail/advice-on-the-use-of-point-of-care-immunodiagnosti c-tests-for-covid-19)." Based on the performance of antigen tests for the influenza virus, the sensitivity of these tests could vary a lot and miss a large number of active infections. The widespread use of these immunodiagnostic tests in clinical settings, in spite of their shortcomings, emphasizes the need for developing rapid and point-of-care (POC) tests that are based on molecular diagnostics (i.e., tests that detect the viral RNA directly in a manner similar to RT-PCR). For an overall understanding of the COVID-19 diagnostic 
landscape, we point readers towards the excellent perspective by Weissleder and colleagues (Weissleder et al. 2020).

\section{Our Past Work in Molecular Diagnostics}

Our research group uses microfluidic technology to develop different healthcare interventions. In the past, we have worked on several molecular diagnostic technologies to detect the genetic material from bacteria on paperfluidic substrates. More specifically, we focussed on detecting DNA from Mycobacterium tuberculosis (MTB), the bacterial pathogen responsible for causing tuberculosis, a respiratory illness widespread in India. Instead of using PCR to amplify DNA, which requires the use of a thermocycler, we used isothermal DNA amplification techniques that can be performed at a single temperature. As a result, a simple hot plate or even a hand-warmer suffices for DNA amplification. We amplified a short sequence of MTB DNA using helicase dependent amplification (HDA) at $65^{\circ} \mathrm{C}$ and in $10 \mathrm{~min}$ on a paper substrate (Shetty et al. 2016). The amplified DNA could be detected either by loading the paper substrate directly into the well of an agarose gel for electrophoresis or by mixing it with a DNA-binding dye (e.g., SYBR Green) and measuring the resulting fluorescence (Fig. 1).

The next challenge in developing a paperfluidic DNA analysis platform was to integrate the sample preparation step into the workflow. It required inactivation of the live pathogenic bacteria, followed by lysing its cell wall to extract the DNA. Our aim was to integrate the sample preparation and DNA amplification steps into a single reaction step without any intermediate DNA purification. We demonstrated an integrated one-tube workflow at $65^{\circ} \mathrm{C}$ and 60 min that completely disinfected the MTB (H37Rv) culture, thermally lysed the bacteria and amplified the DNA by HDA (Shetty et al. 2017) (Fig. 2). We chose thermal lysis to avoid using chemicals that might interfere with the subsequent amplification step. We demonstrated the integrated protocol using the MTB culture in a solution-based reaction.

Finally, we demonstrated the integrated workflow on a paper substrate with Escherichia coli (MG1655) and Mycobacterium smegmatis $\left(\mathrm{mc}^{2} 155\right)$ bacterial cultures by
(A)

$$
\begin{aligned}
& \text { (a) Enclosed } \\
& \text { paper chip }
\end{aligned}
$$

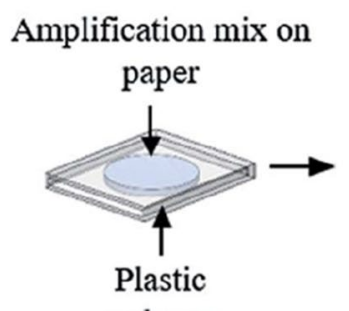

enclosure

(B)

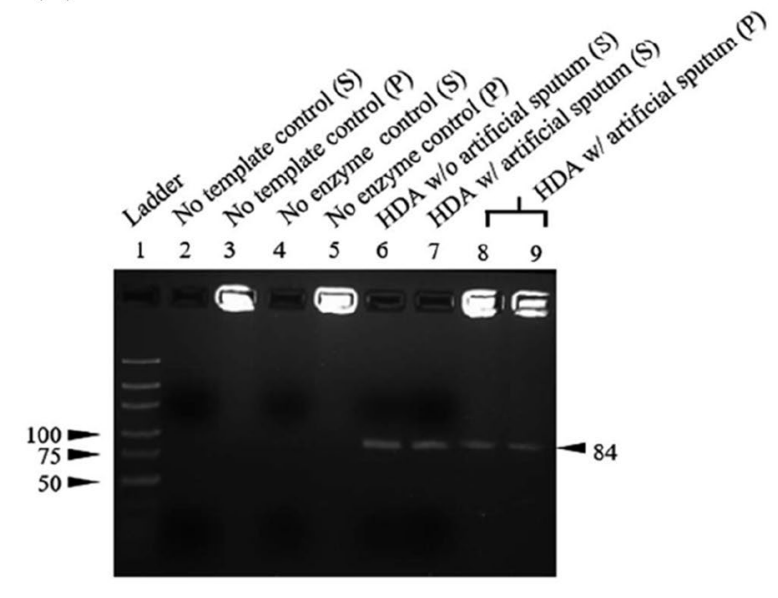

Fig. 1 Amplification of MTB DNA on a paper substrate. a Schematic diagram of our test. b Amplifying TB DNA by HDA in artificial sputum (lanes 8,9$)$. (P) indicates reactions on paper and (S) indicates (b) Helicase dependent amplification

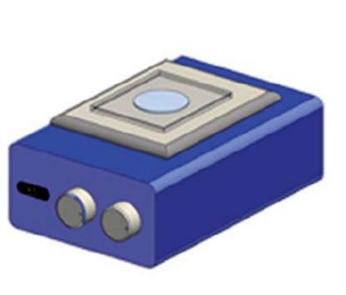

(C)

\section{(c) Fluorescent detection}

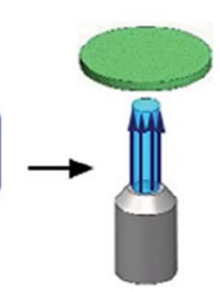

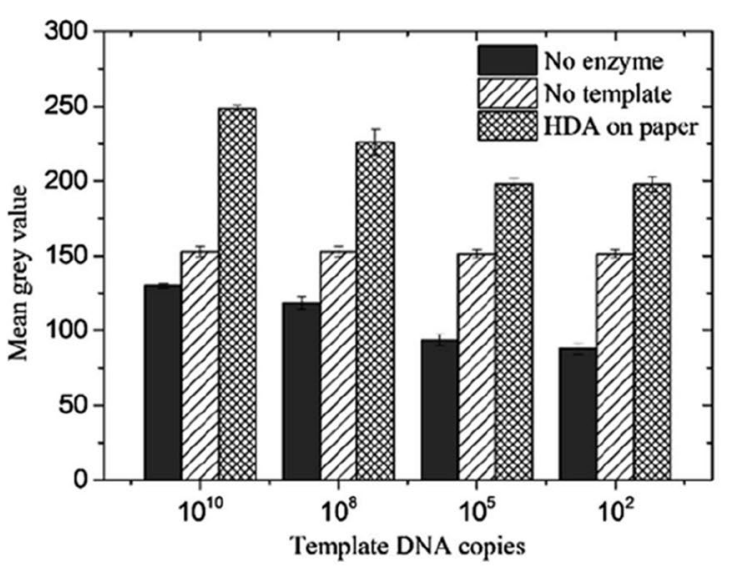

reactions in solution. $\mathbf{c}$ Fluorescence detection of the amplified DNA on paper. Reproduced from Shetty et al. (2016) with permission from Royal Society of Chemistry 
(A)

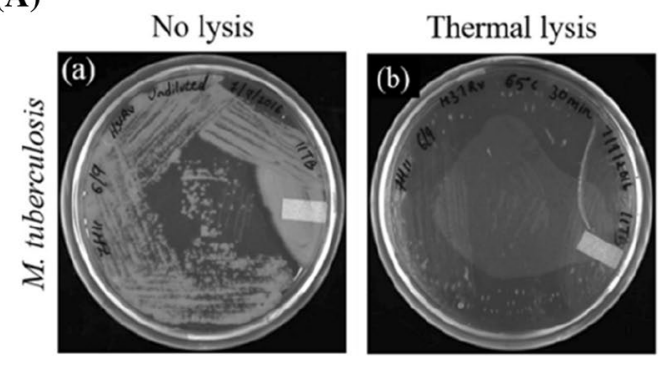

Fig. 2 One-tube integrated thermal lysis and isothermal amplification from MTB (H37Rv) culture. a The thermal lysate was not viable even after 4 weeks of culture. $\mathbf{b}$ Thermal disinfection of pathogenic bacte-

incubating them with the reaction components at $60{ }^{\circ} \mathrm{C}$ for $15 \mathrm{~min}$ and $30 \mathrm{~min}$, respectively (Naik et al. 2019). We switched to a different DNA amplification technique called "loop-mediated isothermal amplification" (LAMP), while optimizing the integrated protocol on a paperfluidic substrate. LAMP is more sensitive compared to HDA as it uses more than one set of primers, and, therefore, is the most common isothermal amplification technique used in paperfluidics. Other than performing DNA analysis on paper substrates, we also have expertise in patterning and developing other kinds of paperfluidic devices (Jagirdar et al. 2015).

\section{What Can We Do to Develop a Diagnostic Test for COVID-19?}

We believe we can build on our past experience with isothermal DNA amplification techniques and paperfluidic devices to develop an isothermal amplification-based molecular diagnostic test for COVID-19 that can be deployed more easily. A literature search reveals that diagnostic tests along similar lines are already under development elsewhere in the world. The ID NOW COVID-19 assay from Abbott, which recently got an emergency use authorization (EUA) from the US government for clinical use, detects SARS-CoV-2 RNA using an isothermal amplification test and can enable a clinical decision in as early as $13 \mathrm{~min}$ (ID NOWTM Covid19 2020). The DETECTR technology, which is under joint development by Mammoth Biosciences and the University of California San Francisco, combines RT-LAMP with CRISPR-based detection in 30 min (Broughton et al. 2019). It uses a paper-based lateral flow assay (LFA) for read-out of the detection signal. The Broad Institute of MIT and Harvard University have recently shared their SHERLOCK protocol
(B)

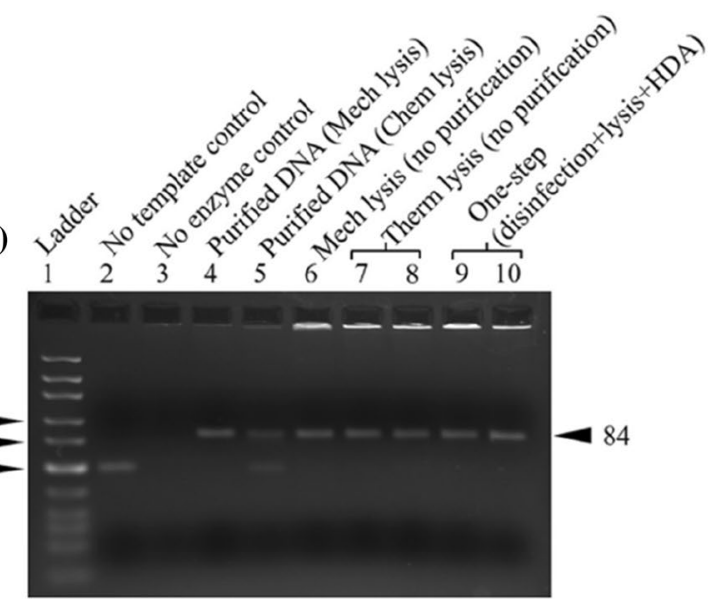

ria, lysis and HDA achieved in a single heat incubation step at $65{ }^{\circ} \mathrm{C}$ and 60 min (lanes 9, 10). Reproduced from Shetty et al. (2017) with permission from Elsevier

using another isothermal technique called "recombinase polymerase amplification" (RPA) in combination with CRISPR (Metsky et al. 2020). While this technology is yet to be validated with clinical samples, it can detect 10 copies of viral RNA per reaction in $60 \mathrm{~min}$. The read-out is again visual, using a paper dipstick. The AIOD-CRISPR platform from the University of Connecticut Health Center also combines RPA and CRISPR to give results in $40 \mathrm{~min}$ (Ding et al. 2020). Like SHERLOCK, this test is for 'research use only' (RUO) until now.

Atila Biosystems have adapted their proprietary isothermal amplification technique OMEGA for detecting SARSCoV-2 RNA in the form of iAMP COVID-19 detection kit (https://www.fda.gov/media/136870). A major advantage of this technique is that it does not require RNA extraction from swabs prior to amplification. This technique has recently been granted a EUA by the US FDA. In India, researchers at the Institute of Genomics and Integrative Biology (IGIB) have recently developed a similar paper-based test that they named 'FnCas9 Editor Linked Uniform Detection Assay' (FELUDA), which is capable of distinguishing single nucleotide variants (Azhar et al. 2020). The authors reported a protocol that can be used for diagnosing COVID-19 from clinical samples using RT-PCR, followed by CRISPR and LFA-based detection on a paper substrate. FELUDA is a promising tool for distinguishing between viruses with similar genomes (e.g., SARS-CoV and SARS-CoV-2).

We propose a molecular test using isothermal DNA amplification for the diagnosis of COVID-19 in India. The typical process flow involves inactivation of the virus, extraction of the RNA, reverse transcription followed by isothermal amplification and detection. We expect the major challenges in this workflow to be (a) effective inactivation of the virus and the RNase, and (b) carrying out RT-LAMP 
in presence of debris without a separate purification process. Chin and others have shown that heat incubation at $70{ }^{\circ} \mathrm{C}$ for 5 min is sufficient to inactivate the virus (Chin et al. 2020). Alternatively, the nasal swab might be directly added to a lysis buffer, thereby inactivating the virus, and brought to the lab for detection. Recently, there were multiple reports on DNA amplification directly from viral swabs without RNA extraction or purification (https://www.fda.gov/media 1136870; Bruce et al. 2020). While LAMP and RPA have both been extensively used, we plan to employ LAMP as it is a more sensitive technique. Existing reports on LAMP have targeted the ORF1a/b, N and RdRP genes of SARS-CoV-2 sequence (https://www.fda.gov/media/136870; Zhang et al. 2020; Mohon et al. 2020; Rabe et al. 2020). As the details of the LAMP primers have already been reported, it will be easier for us to adapt the test in India. The choice of the amplification target and the primers need to be such that we can distinguish between SARS-CoV and SARS-CoV-2. We propose to develop a probe-based lateral flow assay to detect the LAMP reaction products. In the first phase, we would perform all the reactions in solution and perform the detection on a lateral flow device. Given the merits of paperfluidic technology and our experience with it, we believe that it would be useful to develop a complete paperfluidic platform integrating DNA amplification with lateral flow-based detection at a later stage.

\section{Resources and Time to Develop the Diagnostic Test}

Developing an effective diagnostic test requires expertise in different disciplines. We would like to collaborate with other research groups in either academia or industry with expertise in molecular diagnostics technologies. We plan to start by optimizing RT-LAMP using commercially available SARS-CoV-2 RNA samples, followed by the development of a complementary lateral flow strip for detection of the amplified DNA. While we can begin our work with commercially available heat-inactivated SARS-CoV-2 strains, we will need to test this protocol with clinical samples. To this end, we would also like to collaborate with organizations who have access to BSL3 facility, patient samples and experience in handling live viruses. The biggest source of expenditure during the development of the test in the lab is the commercial LAMP kit. Therefore, it would also be very useful to collaborate with an Indian manufacturer of these reagents.

It should be noted that the timeline required for developing molecular POC tests can be as long as 1-2 years. The platform, once validated with a single viral pathogen, can then be adapted more quickly to other viral pathogens. Since we have had outbreaks of several viral diseases (e.g., SARS, MERS, Nipah, etc.) in the recent past, we believe it is extremely important to invest in the development of indigenous rapid point-of-care molecular diagnostic technologies in preparation for future pandemics.

\section{References}

Azhar M et al. (2020) Rapid, field-deployable nucleobase detection and identification using FnCas9. bioRxiv preprint. https://doi. org/10.1101/2020.04.07.028167

Broughton et al. (2020) Rapid detection of 2019 novel coronavirus SARS-CoV-2 using a CRISPR-based DETECTR lateral flow assay. medRxiv preprint. https://doi.org/10.1101/2020.03.06.20032334

Bruce E et al. (2020) RT-qPCR detection of SARS-CoV-2 RNA from patient nasopharyngeal swab using Qiagen RNeasy kits or directly via omission of an RNA extraction step. bioRxiv preprint. https:// doi.org/10.1101/2020.03.20.001008

Chin AWH et al (2020) Stability of SARS-CoV-2 in different environmental conditions. Lancet Microbe 1:e10

Ding et al. (2020) All-in-one dual CRISPR-Cas12a (AIOD-CRISPR) assay: A case for rapid, ultrasensitive and visual detection of novel coronavirus SARS-CoV-2 and HIV virus. bioRxiv preprint. https ://doi.org/10.1101/2020.03.19.998724

ID NOWTM Covid-19. https://www.alere.com/en/home/product-detai ls/id-now-covid-19.html. Accessed 2 Apr 2020

Jagirdar A et al (2015) A paperfluidic device for dental applications using a novel patterning technique. Anal Methods 7:1293

Metsky H et al. (2020) CRISPR-based COVID-19 surveillance using a genomically-comprehensive machine learning approach. bioRxiv preprint. https://doi.org/10.1101/2020.02.26.967026

Mohon AN et al. (2020) Development and validation of direct RT-LAMP for SARS-CoV-2. medRxiv preprint. https://doi. org/10.1101/2020.04.29.20075747

Naik P et al (2019) An integrated one-step assay combining thermal lysis and loop-mediated isothermal DNA amplification (LAMP) in $30 \mathrm{~min}$ from $E$. coli and M. smegmatis cells on a paper substrate. Sens Actuat B 291:74

Rabe B et al. (2020) SARS-CoV-2 detection using an isothermal amplification reaction and a rapid, inexpensive protocol for sample inactivation and purification. medRxiv preprint. https://doi. org/10.1101/2020.04.23.20076877

Shetty P et al (2016) Rapid amplification of Mycobacterium tuberculosis DNA on a paper substrate. RSC Adv 6:56205

Shetty P et al (2017) Thermal lysis and isothermal amplification of Mycobacterium tuberculosis H37Rv in one tube. J Microbiol Methods 143:1

Weissleder $\mathrm{R}$ et al (2020) COVID-19 diagnostics in context. Sci Transl Med 12:eabc1931

Zhang Y et al. (2020) Rapid molecular detection of SARS-CoV-2 (COVID-19) virus RNA using colorimetric LAMP. medRxiv preprint. https://doi.org/10.1101/2020.02.26.20028373

Publisher's Note Springer Nature remains neutral with regard to jurisdictional claims in published maps and institutional affiliations. 